Braz J Med Biol Res, December 2011, Volume 44(12) 1231-1242

doi: 10.1590/S0100-879X2011007500150

Periodic mechanical stress activates MEK1/2-ERK1/2 mitogenic signals in rat chondrocytes through Src and PLC $\gamma 1$

Kewei Ren, Yimin Ma, Yumin Huang, Wenwei Liang, Feng Liu, Qing Wang, Weiding Cui, Zhengyu Liu, Guoyong Yin and Weimin Fan

The Brazilian Journal of Medical and Biological Research is partially financed by

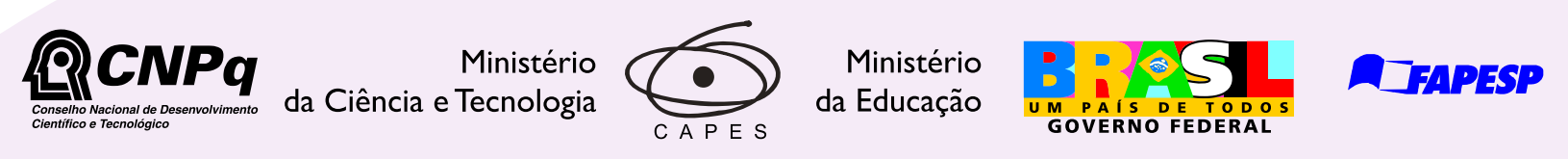

Institutional Sponsors
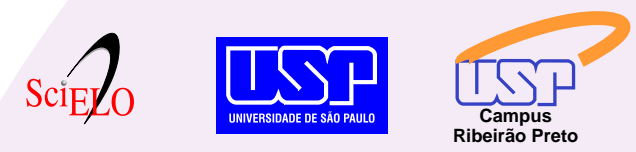

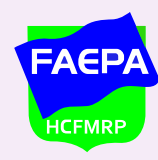

Ф SHIMADZU

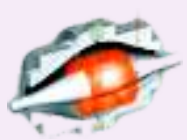

Explore High - Performance MS Orbitrap Technology In Proteomics \& Metabolomics

$\underset{\text { analitica }}{\text { analiticaweb.com.br }}$ SCIENTIFIC 


\title{
Periodic mechanical stress activates MEK1/2-ERK1/2 mitogenic signals in rat chondrocytes through Src and PLCY1
}

Kewei Ren*, Yimin Ma*, Yumin Huang, Wenwei Liang, Feng Liu, Qing Wang, Weiding Cui, Zhengyu Liu, Guoyong Yin and Weimin Fan

Department of Orthopedics, the First Affiliated Hospital, Nanjing Medical University, Nanjing, China

\begin{abstract}
The mitogenic effects of periodic mechanical stress on chondrocytes have been studied extensively but the mechanisms whereby chondrocytes sense and respond to periodic mechanical stress remain a matter of debate. We explored the signal transduction pathways of chondrocyte proliferation and matrix synthesis under periodic mechanical stress. In particular, we sought to identify the role of the MEK1/2-ERK1/2 signaling pathway in chondrocyte proliferation and matrix synthesis following cyclic physiologic mechanical compression. Under periodic mechanical stress, both rat chondrocyte proliferation and matrix synthesis were significantly increased $(P<0.05)$ and were associated with increases in the phosphorylation of Src, PLCY1, MEK1/2, and ERK1/2 $(P<0.05)$. Pretreatment with the MEK1/2-ERK1/2 selective inhibitor, PD98059, and shRNA targeted to ERK1/2 reduced periodic mechanical stress-induced chondrocyte proliferation and matrix synthesis $(P<0.05)$, while the phosphorylation levels of Src-Tyr ${ }^{418}$ and PLCY1-Tyr ${ }^{783}$ were not inhibited. Proliferation, matrix synthesis and phosphorylation of MEK1/2-Ser $217 / 221$ and ERK1/2-Thr202/Tyr204 were inhibited after pretreatment with the PLCY1 inhibitor U73122 in chondrocytes in response to periodic mechanical stress $(P<0.05)$, while the phosphorylation site of Src-Tyr ${ }^{418}$ was not affected. Inhibition of Src activity with PP2 and shRNA targeted to Src abrogated chondrocyte proliferation and matrix synthesis (P $<0.05)$ and attenuated PLCY1, MEK1/2 and ERK1/2 activation in chondrocytes subjected to periodic mechanical stress $(P$ $<0.05)$. These findings suggest that periodic mechanical stress promotes chondrocyte proliferation and matrix synthesis in part through the Src-PLCY1-MEK1/2-ERK1/2 signaling pathway, which links these three important signaling molecules into a mitogenic cascade.
\end{abstract}

Key words: Periodic mechanical stress; Chondrocyte proliferation and matrix synthesis; Src; PLCY1; MEK1/2-ERK1/2

\section{Introduction}

Articular cartilage is normally subjected to a wide variety of complex physical forces (1). These mechanical stimuli are important factors in the maintenance of normal structure and function of the articular cartilage. Periodic mechanical stress, as a special type of mechanical stimulation, can be used to advantage to simulate in vivo physiological mechanical conditions, enhancing the quality of tissue-engineered cartilage by promoting chondrocyte proliferation, migration, and matrix synthesis, which plays an important role in the repair of articular cartilage injury (2-5). However, the mechanisms involved in chondrocytes to detect and respond to periodic mechanical stress have not been identified.

MEK1/2-ERK1/2 is the core component of the MAPK cascade. MEK1/2 and ERK1/2 exist in virtually all eukaryotic cells and control fundamental cellular functions (6-8). There is increasing evidence that MEK and ERK1/2 are involved in the regulation of chondrocyte proliferation, matrix synthesis, and migration in response to periodic mechanical stimulation (9-12). Hung et al. (10) found that MEK-ERK1/2 participates in fluid flow-induced chondrocyte mechanotransduction, leading to regulation of expression of the aggrecan gene, and Perera et al. (13) demonstrated that activated ERK1/2 signaling is required for the mitogenic response of human chondrocytes to cyclic mechanical strain. A previous study by our group has also confirmed that periodic mechanical stress promotes rat chondrocyte area expansion and migra-

Correspondence: Weimin Fan, Department of Orthopedics, the First Affiliated Hospital, Nanjing Medical University, Nanjing 210029, China. E-mail: fanweimin@vip.sina.com

*These authors contributed equally to this study.

Received February 25, 2011. Accepted October 25, 2011. Available online November 11, 2011. Published November $28,2011$. 
tion through the ERK1/2 signaling pathway (12).

The MEK1/2-ERK1/2 signal is regulated by many kinases (14-16); however, the upstream mediators of MEK1/2-ERK1/2, which are responsible for periodic mechanical stimulation-induced chondrocyte proliferation and matrix synthesis, have not been completely characterized. It is universally accepted that the same stimuli may initiate different signaling transduction pathways, which modulate different cellular responses. Indeed, these different signaling transduction pathways initiated by the same stimuli can also converge upon some signaling molecules, such as ERK1/2, mediating different cellular responses $(17,18)$. Our previous study demonstrated that periodic mechanical stress promotes chondrocyte area expansion and migration through the Src-PLCy1-ERK1/2 signaling pathway (12). Thus, one concern is whether or not cyclic mechanical stimulation modulates chondrocyte proliferation and matrix synthesis through the Src-PLCY1-MEK1/2-ERK1/2 signaling pathway as well.

PLCY1, as one of the serine threonine kinases of PLC, plays an important role in the signal transduction for the regulation of the cellular mitogenic effects in various types of cells in response to different stimuli, including cyclic mechanical stimulation (19-22). Liu et al. (22) have demonstrated that intermittent mechanical strain enhances the cellular proliferation and phosphorylation of PLCY1 in fetal lung cells, and the mitogenic response to cyclic mechanical stimulation is mediated by activated PLCY1. Src, which shows the activity of tyrosine kinase, has been shown by many investigators to be required for proliferation in some cell types in response to mechanical stimuli $(14,23)$. Chaturvedi et al. (24) reported that repeated mechanical strain can promote human $\mathrm{H} 441$ pulmonary epithelial cell proliferation and activate Src, while periodic mechanical stimuli-induced mitogenicity depends on the activity of Src. Moreover, it has been reported that Src is involved in regulating the activation of chondrocytes (25).

Thus, we sought to explore the role of the MEK1/2ERK1/2 signaling pathway in chondrocyte proliferation and matrix synthesis following cyclic physiological mechanical stress. In particular, we determined whether or not MEK1/2ERK1/2 signaling and its upstream kinases, Src and PLCY1, mediate the mitogenic effects of periodic mechanical stress on chondrocytes. Therefore, we speculate that periodic mechanical stress promotes chondrocyte proliferation and matrix synthesis through the Src-PLCY1-MEK1/2-ERK1/2 signaling pathway.

\section{Material and Methods}

\section{Material}

Two-week-old Sprague-Dawley (SD) rats of either sex were provided by the Animal Center of Nanjing Medical University. Fetal bovine serum was purchased from Hangzhou Sijiqing Biological Engineering Materials Co., Ltd. (China).
DMEM-F12, trypsin, collagenase II, and anti-collagen monoclonal antibody II were purchased from Gibco (USA). Type II collagen (Col2) was purchased from Sigma (USA). Cell counting kit-8 (CCK-8) was purchased from Beyotime Institute of Biotechnology (China). Anti-Src, anti-phosphoSrc (Tyr $\left.{ }^{418}\right)$, anti-PLCY1, anti-phospho-PLCY1 (Tyr $\left.{ }^{783}\right)$, anti-ERK1/2, anti-phospho-ERK1/2 (Thr $\left.202 / \mathrm{Tyr}^{204}\right)$, horseradish peroxidase (HRP)-goat anti-rabbit IgG, and PD98059 were supplied by CST Co., Ltd. (USA). Electrochemiluminescence (ECL), PP2 and U73122 were purchased from Amersham (UK), BIOMOL (USA), and Santa Cruz (USA), respectively. RNAiso Plus, PrimeScript RT reagent kit, and SYBR Premix Ex Taq II were purchased from TaKaRa (Japan). Src shRNA (r) Lentiviral Particles, ERK1 shRNA (r) Lentiviral Particles, ERK2 shRNA ( $r$ ) Lentiviral Particles, Control shRNA Lentiviral Particles, and Polybrene were supplied by Santa Cruz.

A cell incubator (Hereus BB 5060), an air-tight cell culture device and reciprocating pressure pump, a barrier type pressure transducer and inversion microscope equipped with a camera system were purchased from Hereus (Germany), Taixing Experimental Instrument Factory (China), Tianjin Plastics Research Institute (China), and Olympus (Japan), respectively.

\section{Methods}

Cell culture. Chondrocytes were harvested by the method described by Séguin and Bernier (26). Briefly, under sterile conditions, cartilage derived from the limb joints of 2-week-old SD rats was harvested and chopped into fragments of approximately $1 \mathrm{~mm}^{3}$. The tissues were digested with $0.25 \%$ trypsin at $37^{\circ} \mathrm{C}$ for $0.5 \mathrm{~h}$, followed by digestion with $0.2 \%$ collagenase $\mathrm{Il}$ at $37^{\circ} \mathrm{C}$ for $4 \mathrm{~h}$. Cells were harvested by filtering with a 200 -mesh filter and cultured in DMEM-F12 culture medium supplemented with $10 \%$ FBS at $37^{\circ} \mathrm{C}$ with $5 \% \mathrm{CO}_{2}$ in an incubator. Cells were purified by repeated adherence, and morphology was observed under an inverted phase contrast microscope by staining Col2 according to the conventional ABC method. Second generation cells were seeded on a glass slide $(25 \times 25 \mathrm{~mm})$ coated with Col 2 at a density of $10^{5}$ cells. Experiments were performed when cells had reached approximately $70-80 \%$ confluence.

Inhibitors. PP2, U73122 and PD98059 were specific inhibitors of Src, PLCY1 and ERK1/2, respectively. Each inhibitor was dissolved in anhydrous dimethylsulfoxide (DMSO) to form a concentrated solution that was 1000 times the required final concentration. All inhibitors were aliquoted and stored at $-20^{\circ} \mathrm{C}$. Each concentrated solution was diluted $1000 \mathrm{X}$ immediately prior to use and each pretreatment group contained $0.1 \%(\mathrm{v} / \mathrm{v}) \mathrm{DMSO}$, which was the concentration used for the control group. Cells were pretreated with $10 \mu \mathrm{M}$ PP2, $10 \mu \mathrm{M}$ U73122, $50 \mu \mathrm{M}$ PD98059, or an equivalent amount of DMSO $(0.1 \%, v / v)$ for $30 \mathrm{~min}$, respectively. 
Construction of a periodic mechanical stress field. A periodic stress field of the perfusion culture system with adjustable stress intensity and frequency was built by connecting the reciprocating intensifier pump to the air-tight cell culture device through a barrier type pressure transducer, as previously described (12). The pressure in this system ranged from 0 to $300 \mathrm{kPa}$ and the frequency from 0 to 1 $\mathrm{Hz}$. We previously found that tissue-engineered cartilage exhibited the best quality when the rabbit chondrocytes underwent stress varying from 0 to $200 \mathrm{kPa}$ at $0.1 \mathrm{~Hz}(27,28)$. Therefore, this pressure range $(0-200 \mathrm{kPa})$ and frequency $(0.1 \mathrm{~Hz})$ were used in all experiments.

Experimental grouping. The experiment consisted of two steps. The first step was to explore the effects of periodic mechanical stress on cell proliferation, matrix synthesis as well as the expression and phosphorylation of Src, PLCY1, MEK1/2, and ERK1/2. Cells were divided into a non-pressure group and a pressure group and incubated in a simple-cell culture device and under a periodic mechanical stress field, respectively. Cells were maintained under static conditions or periodic mechanical stress for $0,0.5,1$, and $2 \mathrm{~h}$ prior to Western blot analysis. Cells were maintained under static conditions or periodic mechanical stress for 8 h prior to quantitative real-time PCR analysis, respectively. Cells were cultured for 3 days under static conditions or periodic mechanical stress $8 \mathrm{~h}$ per day prior to direct cell counting and CCK-8 assay, respectively.

The second step was to explore the effects of MEK1/2ERK1/2, PLCy1, and Src on cell proliferation, matrix synthesis and the expression and nature of the relationship between Src, PLCY1, MEK1/2, and ERK1/2 under conditions of periodic mechanical stress. For this step, chondrocytes were treated with $10 \mu \mathrm{M}$ PP2 (PP2 group), $10 \mu \mathrm{M}$ U73122 (U73122 group), 50 M PD98059 (PD98059 group), or an equivalent amount of DMSO $(0.1 \%, v / v$, control group) for a 30-min period of pretreatment, or chondrocytes were pretreated with shRNA targeted to Src and ERK1/2 or control scrambled shRNA, respectively. Cells were then incubated under static conditions or periodic mechanical stress for $1 \mathrm{~h}$ prior to Western blot analysis and for $8 \mathrm{~h}$ prior to quantitative real-time PCR analysis, respectively. Cells were cultured for 3 days under static conditions or periodic mechanical stress $8 \mathrm{~h}$ per day prior to direct cell counting and CCK-8 assay, respectively. In all experiments, all cell groups were incubated at $37^{\circ} \mathrm{C}$ in the presence of $5 \% \mathrm{CO}_{2}$.

Western blot analysis. Total protein was prepared using RIPA buffer, and Western blot analyses were performed as previously described (29). Briefly, cells were rinsed with PBS and lysed using a cell lysate (50 mM Tris, $\mathrm{pH} 7.4$, $150 \mathrm{mM} \mathrm{NaCl}, 1 \% \mathrm{NP}-40,0.25 \%$ sodium deoxycholate, $1 \mathrm{mM}$ EDTA, $1 \mathrm{mM}$ NaF, $1 \mathrm{mM}$ phenylmethylsulfonyl fluoride (PMSF), $1 \mathrm{mM}$ aprotinin, and $1 \mathrm{mM}$ $\mathrm{Na}_{3} \mathrm{VO}_{4}$ ). Homogenates were clarified by centrifugation $(15,805 \mathrm{~g})$ for $10 \mathrm{~min}$ at $4^{\circ} \mathrm{C}$, and the supernatants were removed and analyzed for protein concentration by the method of Bradford. Protein mixtures were separated by SDS-PAGE and transferred to nitrocellulose membranes. Following blocking for $1 \mathrm{~h}$ with $5 \%$ milk in Tris-buffered saline and Tween 20 (TBST), membranes were incubated with anti-phospho-Src (Tyr $\left.{ }^{418}\right)$, anti-phospho-PLCY1 (Tyr $\left.{ }^{783}\right)$,

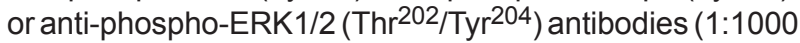
dilution for all three antibodies) overnight at $4^{\circ} \mathrm{C}$. Blots were then incubated with $\mathrm{HRP}$-conjugated secondary antibody at room temperature for $1 \mathrm{~h}$ and detected by ECL. The results were scanned with the Gel Imaging System (UVP Company) and measured with the Gel-Pro Analyzer software (Media Cybernetics).

Quantitative real-time PCR analysis. Total RNA was extracted with RNAiso Plus according to the manufacturer protocol and eluted in PCR-grade water and the collected samples were stored at $-80^{\circ} \mathrm{C}$. RNA was reverse transcribed into cDNA with the PrimeScript RT reagent kit according to the manufacturer protocol. cDNA was eluted in PCRgrade water and stored at $-30^{\circ} \mathrm{C}$. Quantitative real-time PCR analysis was performed with the LightCycler System (Roche Diagnostics) using SYBR Premix Ex Taq II according to the manufacturer protocol. The reaction was performed in a $20-\mu \mathrm{L}$ mixture containing $2 \mu \mathrm{L}$ of the above cDNA. For aggrecan (AGC), Col2, and glyceraldehyde-3-phosphate dehydrogenase (GAPDH), each cDNAsample was amplified using specific primers (TaKaRa; Table 1). The cycling conditions were a 30 -s initial denaturation step at $95^{\circ} \mathrm{C}$ followed by 40 cycles at $95^{\circ} \mathrm{C}$ for $5 \mathrm{~s}$, and at $60^{\circ} \mathrm{C}$ for $20 \mathrm{~s}$. To normalize mRNA levels, we amplified the GAPDH housekeeping gene as an internal control. Gene expression for AGC and Col2 was normalized against that for GAPDH.

Proliferation studies. Proliferation studies were assessed by two different methods, i.e., direct cell counting and CCK-8 assay.

Direct cell counting. Second generation chrondrocytes

Table 1. Primer sequences and product sizes used in real-time PCR.

\begin{tabular}{llcc}
\hline Genes & Sequence of primers (5'-3') & AS (bp) & AT $\left({ }^{\circ} \mathrm{C}\right)$ \\
\hline Aggrecan & F: GAAGTGATGCATGGCATTGAGG & 146 & 60 \\
& R: ATGATGGCGCTGTTCTGAAGG & & \\
Type II collagen & F: GAGGGCAACAGCAGGTTCAC & 95 & 60 \\
& R: TGTGATCGGTACTCGATGATGG & & \\
GAPDH & F: GGCACAGTCAAGGCTGAGAATG & 143 & 60 \\
& R: ATGGTGGTGAAGACGCCAGTA & & \\
\hline
\end{tabular}

$\mathrm{F}$ = forward; $\mathrm{R}$ = reverse; $\mathrm{AS}=$ amplicon size; $\mathrm{bp}$ = base pairs; $\mathrm{AT}$ = annealing temperature; GAPDH = glyceraldehyde-3-phosphate dehydrogenase. 
were seeded on glass slides $(25 \times 25 \mathrm{~mm})$ coated with Col2 at a density of $10^{5}$ cells and were randomly divided into different groups. Each group included cells on six glass slides. Experiments were performed when cells had reached approximately $70-80 \%$ confluence. Chondrocytes were cultured for 3 days under non-pressure conditions or conditions of periodic mechanical stress $8 \mathrm{~h}$ per day prior to direct cell counting. Cells were trypsinized and counted. Cell number was determined by counting cells from each glass slide independently. The experiments were repeated five times.

CCK-8 assay. Chondrocytes were cultured for 3 days under non-pressure conditions or conditions of periodic mechanical stress $8 \mathrm{~h}$ per day prior to CCK-8 assay. Cell proliferation was determined using a CCK-8 solution according to manufacturer instructions. Twenty microliters CCK-8 solution was added to cells in each well of five 96well plates and incubated for $1 \mathrm{~h}$ at $37^{\circ} \mathrm{C}$. The absorbance of each well was finally determined at $450 \mathrm{~nm}$ using a microplate reader.

\section{Statistical analysis}

Statistical analyses were performed using the SPSS
14.0 software and data are reported as means \pm SD. Student unpaired $t$-tests were used to determine the statistical significance. A P value of 0.05 was considered to be significant.

\section{Results}

\section{Effects of periodic mechanical stress on cell proliferation and matrix synthesis}

Cell proliferation in the pressure group was increased compared to the non-pressure group $(P<0.05$, Figure 1 , $N=5$ ). Quantitative real-time PCR showed that AGC and Col2 gene expression were up-regulated in the pressure group compared to the non-pressure group $(P<0.05$, Figure 1, $\mathrm{N}=5$ ).

\section{Effects of periodic mechanical stress on the expression and phosphorylation of Src, PLCY1, MEK1/2, and ERK1/2}

Western blot analysis revealed that the phosphorylation levels of Src, PLCY1, MEK1/2, and ERK1/2 at $0.5 \mathrm{~h}, 1 \mathrm{~h}$ and $2 \mathrm{~h}$ were increased in the pressure group compared to the non-pressure group $(P<0.05$, Figure $2, N=5)$.
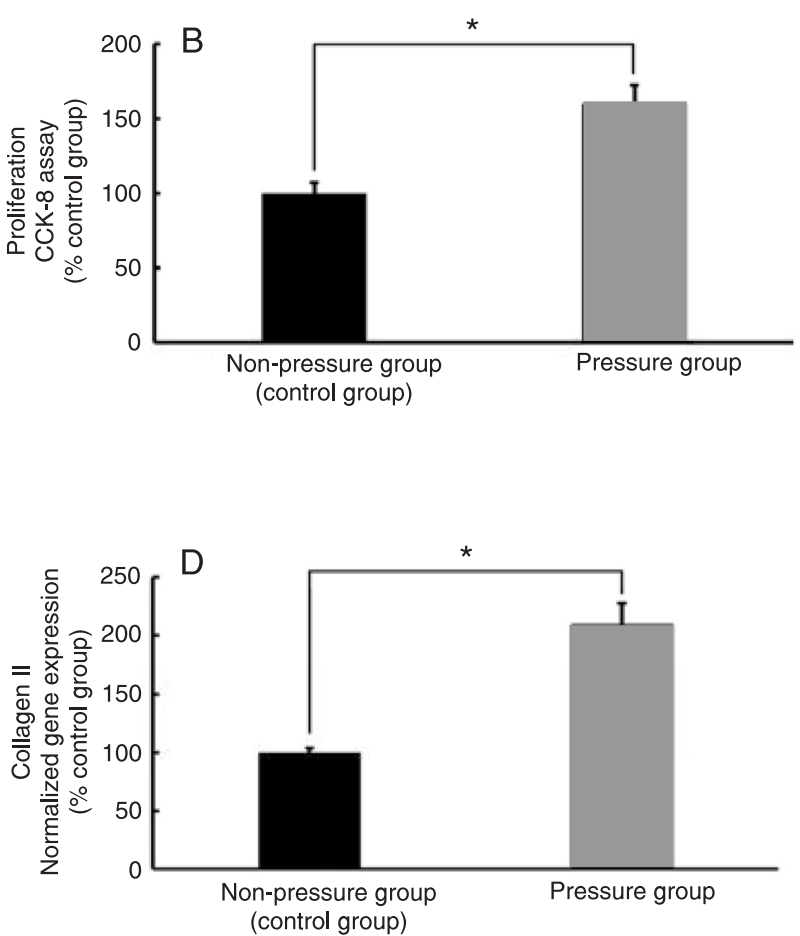

Figure 1. Effect of periodic mechanical stress on chondrocyte proliferation and matrix synthesis. Rat chondrocytes were cultured for 3 days under static conditions or conditions of periodic mechanical stress $8 \mathrm{~h}$ per day prior to proliferation studies. Cell proliferation was analyzed using direct cell counting $(A)$ and a CCK-8 assay $(B)$. Chondrocytes were cultured in vitro for $8 \mathrm{~h}$, with or without periodic mechanical stress prior to matrix synthesis studies. Aggrecan $(C)$ and type II collagen $(D)$ gene expression were measured to indicate the level of matrix synthesis, which was measured by quantitative real-time PCR. Chondrocyte proliferation and matrix synthesis in the pressure group were significantly increased relative to that in the non-pressure group $\left(\mathrm{N}=5,{ }^{*} \mathrm{P}<0.05\right.$, Student unpaired $t$-test). 

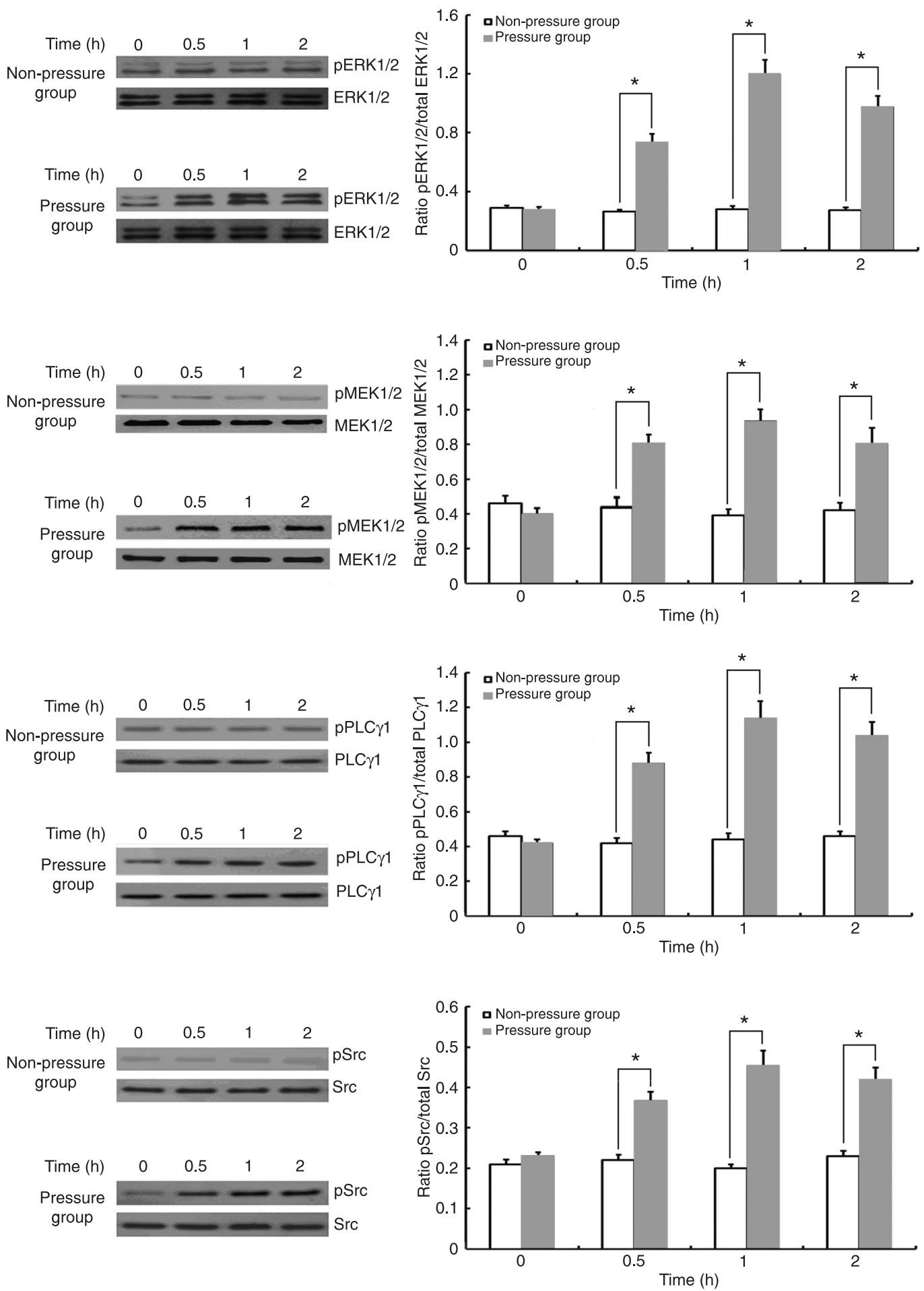

Figure 2. Effect of periodic mechanical stress on the expression and phosphorylation of ERK1/2, MEK, PLCY1, and Src. Rat chondrocytes were cultured in vitro for $0,0.5,1$, and $2 \mathrm{~h}$, with or without periodic mechanical stress. The expression and phosphorylation level of ERK1/2, MEK, PLCY1, and Src were detected by Western blotting, and the total quantity of each protein served as the control. The images on the left are representative of the results of Western blotting. The phosphorylation levels of ERK1/2-Thr $202 / \mathrm{Tyr}^{204}$, MEK-Ser ${ }^{217 / 221}$, PLCY1-Tyr ${ }^{783}$, and Src-Tyr ${ }^{418}$ in the pressure group at $0.5,1$ and $2 \mathrm{~h}$ were significantly increased relative to those in the non-pressure group $(\mathrm{N}$ $=5,{ }^{*} \mathrm{P}<0.05$, Student unpaired $t$-test). 
Effects of MEK1/2-ERK1/2, PLCY1, and Src on cell proliferation and matrix synthesis under conditions of periodic mechanical stress

Cell proliferation in the inhibitor groups and shRNA groups was significantly reduced compared to control in response to periodic mechanical stress $(P<0.05$, Figures 3 and $4, N=5)$. At the same time, compared with the control groups, AGC and Col2 gene expression in the inhibitor groups and shRNA groups subjected to periodic mechanical stress were significantly attenuated $(P<0.05$, Figures 3 and $4, N=5$ ).

Nature of the relationship between Src, PLC, MEK1/2, and ERK1/2 under conditions of periodic mechanical stress

The phosphorylation levels of Src and PLCY1 were not affected in the PD98059 and ERK1/2 shRNA groups compared to control $(P>0.05, N=5)$.

A
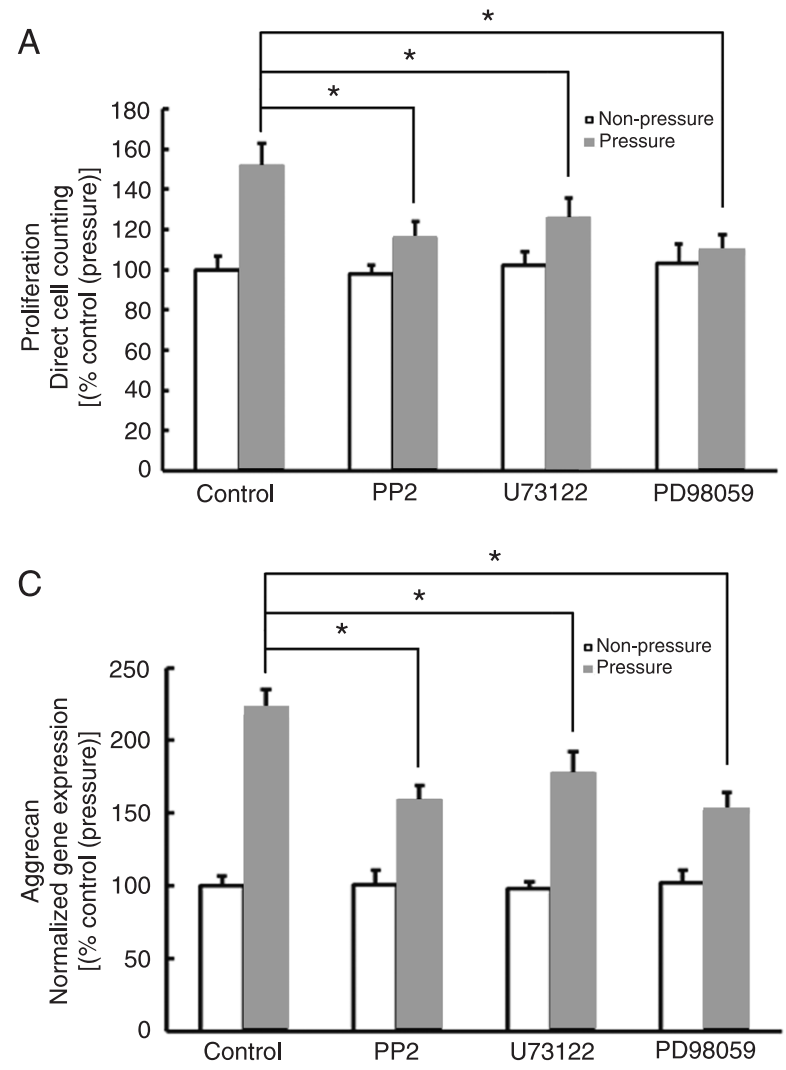

The phosphorylation levels of MEK1/2 and ERK1/2 in the U73122 group were significantly decreased relative to those in the control groups $(P<0.05$, Figure $5, N=5)$, but the phosphorylation level of $\mathrm{Src}$ was not altered $(P>0.05$, Figure 5, N = 5).

The phosphorylation levels of PLCY1, MEK1/2, and ERK $1 / 2$ in the PP2 and Src shRNA groups were significantly blocked when compared to those of the control groups ( $P$ $<0.05$, Figure $6, N=5$ ).

\section{Discussion}

The present study was designed to determine the mechanism of signal transduction by which periodic mechanical stress promotes chondrocyte proliferation and matrix synthesis. In particular, we sought to identify whether or not Src, PLCY1, MEK $1 / 2$, and ERK $1 / 2$ are involved in regulating the mitogenic response to cyclic mechanical stimulation

B
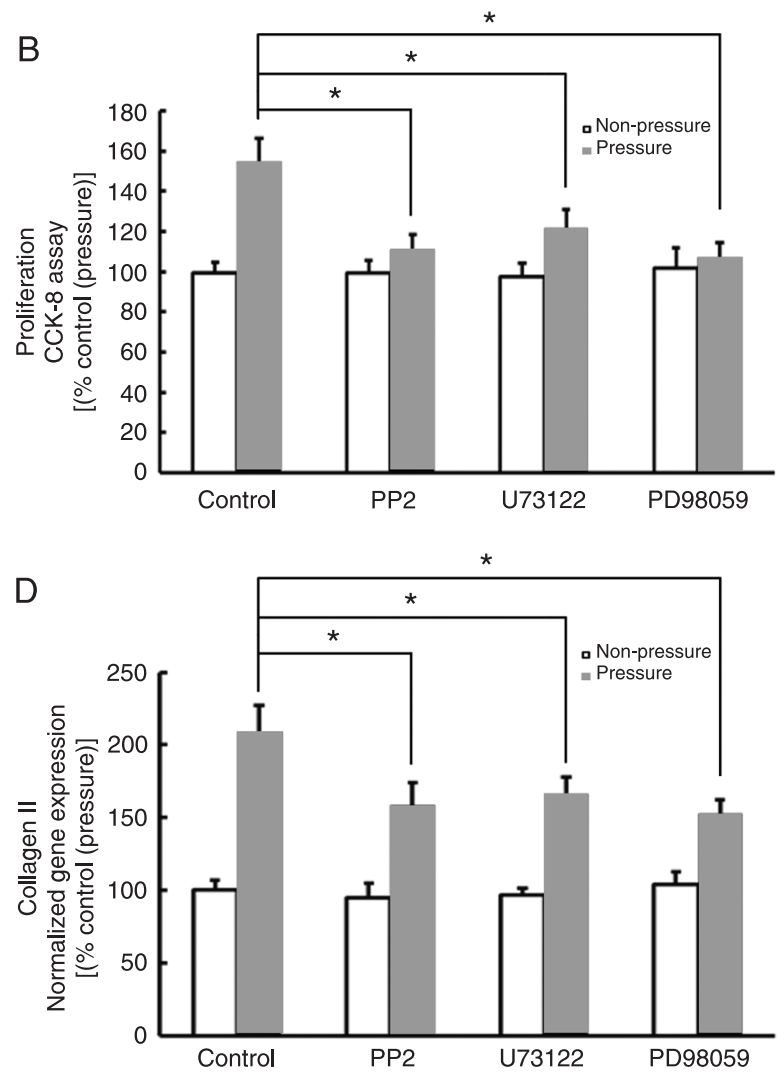

Figure 3. PP2, U73122 and PD98059 inhibit periodic mechanical stress-induced cell proliferation and matrix synthesis. After pretreatment with DMSO or ERK1/2 inhibitor (PD98059), PLCY1 inhibitor (U73122) and Src inhibitor (PP2), rat chondrocytes were cultured for 3 days under static conditions or conditions of periodic mechanical stress $8 \mathrm{~h}$ per day prior to proliferation studies. Cell proliferation was analyzed by direct cell counting $(A)$ and CCK-8 assay $(B)$. Chondrocytes were cultured in vitro for $8 \mathrm{~h}$ under static conditions or periodic mechanical stress prior to matrix synthesis studies. Aggrecan $(C)$ and type II collagen $(D)$ gene expression were measured to indicate the level of matrix synthesis, which was measured by quantitative real-time PCR. Chondrocyte proliferation and matrix synthesis in the inhibitor pretreatment groups was significantly decreased relative to control in chondrocytes in response to periodic mechanical stress $\left(\mathrm{N}=5,{ }^{*} \mathrm{P}<0.05\right.$, Student unpaired $t$-test). 
A
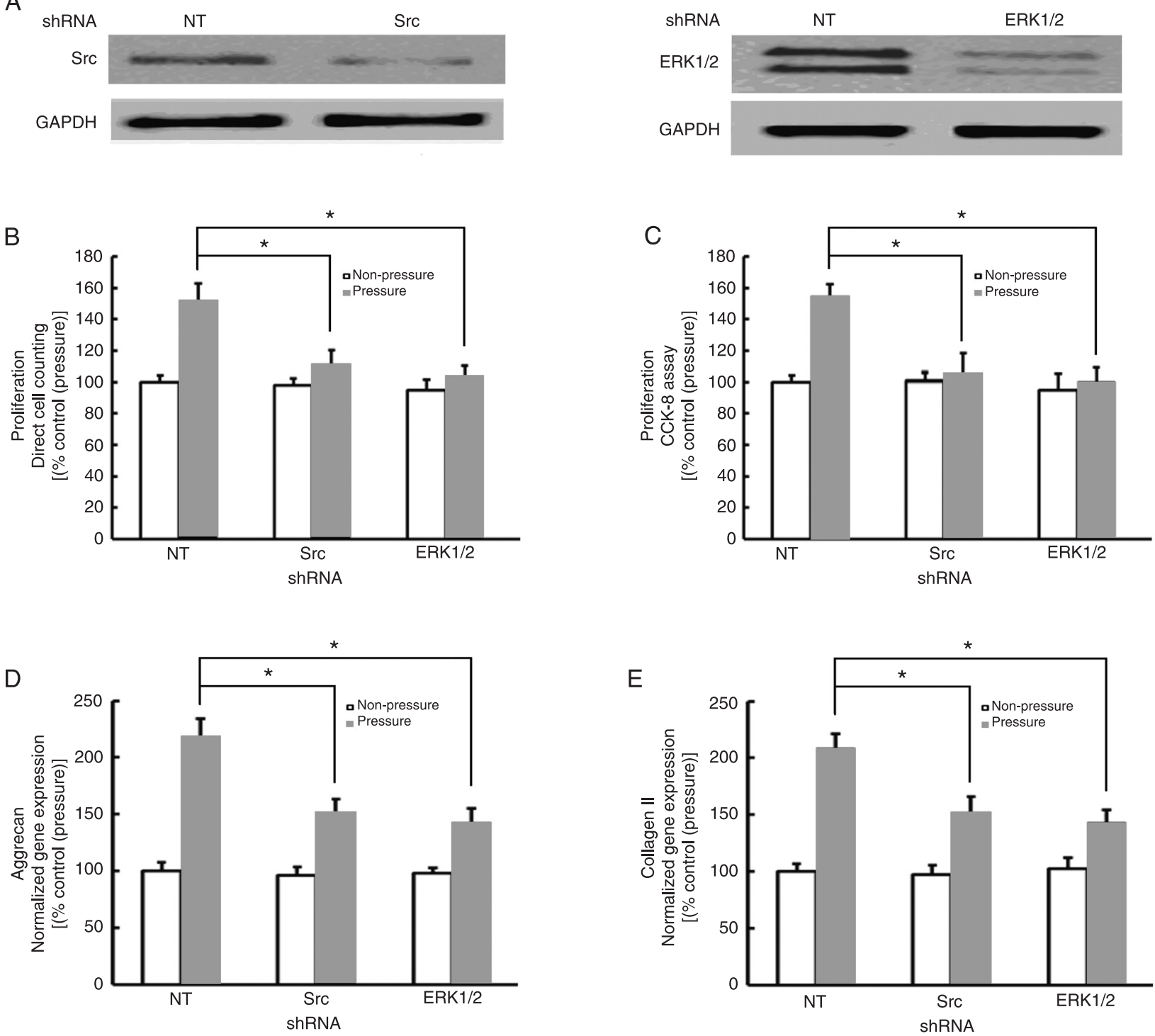

Figure 4. Src shRNA and ERK1/2 shRNA inhibit periodic mechanical stress-induced chondrocyte proliferation and matrix synthesis. $A$, Chondrocytes were transfected with shRNA targeted to Src or ERK1/2 or with non-targeting (NT) sequences prior to lysis and Western blot for Src and ERK1/2 protein. Either the Src or ERK1/2 shRNA sequence achieved about a 50\% reduction in Src or ERK1/2 protein levels. After pretreatment with control shRNA or Src shRNA and ERK1/2 shRNA, rat chondrocytes were cultured for 3 days under static conditions or conditions of periodic mechanical stress $8 \mathrm{~h}$ per day prior to proliferation studies. Cell proliferation was analyzed by direct cell counting $(B)$ and CCK-8 assay $(C)$. Rat chondrocytes were cultured in vitro for $8 \mathrm{~h}$ with static conditions or periodic mechanical stress. Aggrecan $(D)$ and type II collagen $(E)$ gene expression were measured by quantitative real-time PCR. Cell proliferation and matrix synthesis in the inhibitor pretreatment groups was significantly decreased relative to control in chondrocytes in response to periodic mechanical stress $\left(\mathrm{N}=5,{ }^{*} \mathrm{P}<0.05\right.$, Student unpaired $t$-test $)$.

in chondrocytes. The present data suggest that Src and PLCY1 may mediate cyclic mechanical strain-induced proliferation and matrix synthesis in chondrocytes by regulating MEK1/2-ERK1/2 phosphorylation. This conclusion is based on the observations that periodic mechanical stress of chondrocytes induced proliferation, matrix synthesis, and ERK1/2 activation and that these effects were inhibited by inhibiting Src, PLCY1, MEK1/2, or ERK1/2.

MEK1/2-ERK $1 / 2$ is initiated in response to numerous stimuli from the micro-environment of cells $(30,31)$. MEK1/2-ERK1/2 activation has been linked to mechanical stimulation-induced proliferation in diverse cell types, including chondrocytes $(13,24,32)$. MEK1/2 activated ERK1/2, which functions as a final effector of signal transduction 
pathways and plays an essential role in cell proliferation, differentiation, and migration by directly activating transcription factors in the cytoplasm and nucleus $(6,7,33)$. Ryan et al. (9) reported that cyclic mechanical compression of articular cartilage induces chondrocyte proliferation by activation of the ERK1/2 pathway. As the immediately upstream regulatory kinase of ERK1/2, pretreatment with the selective MEK inhibitor PD98059 could down-regulate the
A

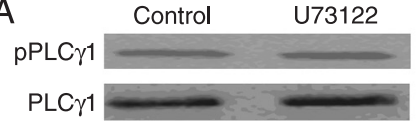

Non-pressure

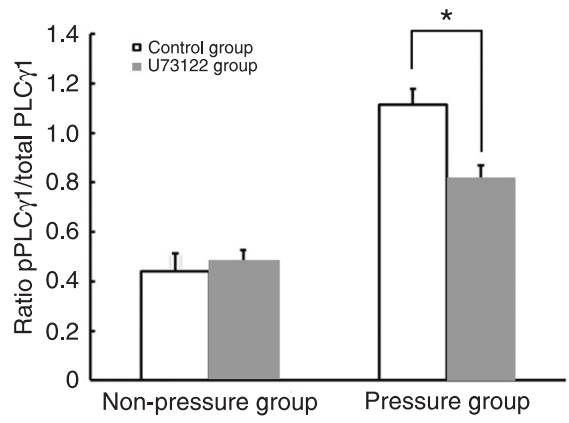

C

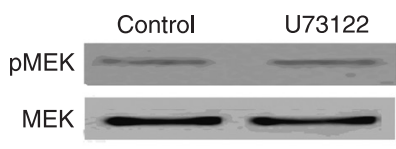

Non-pressure

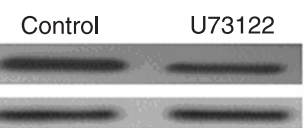

Pressure

B

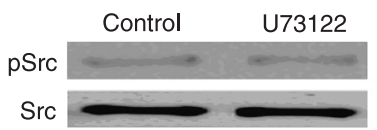

Non-pressure

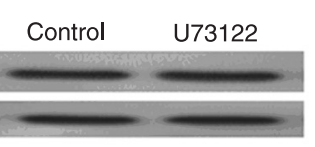

Pressure

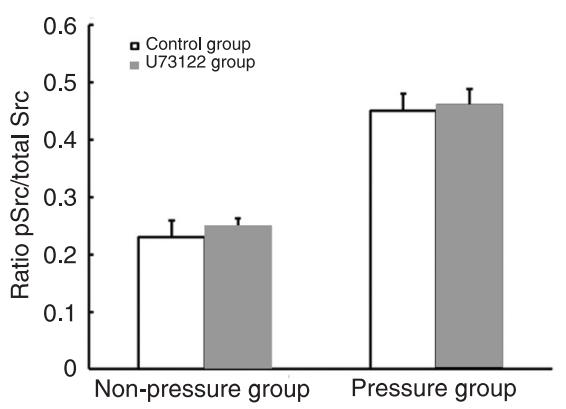

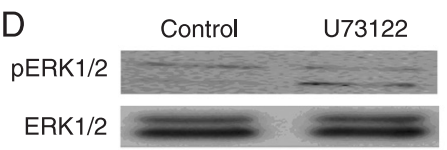

Non-pressure

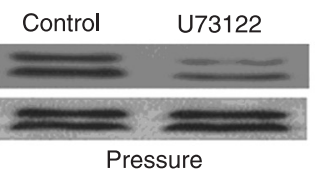

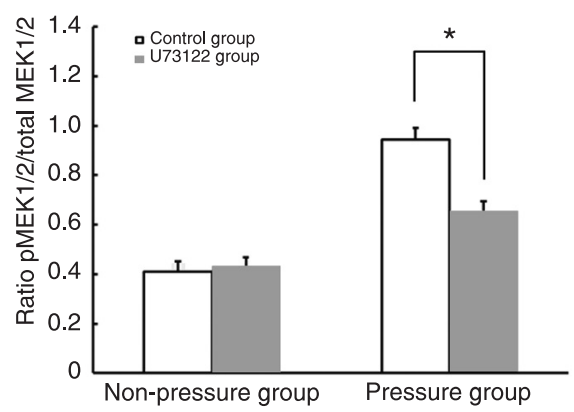

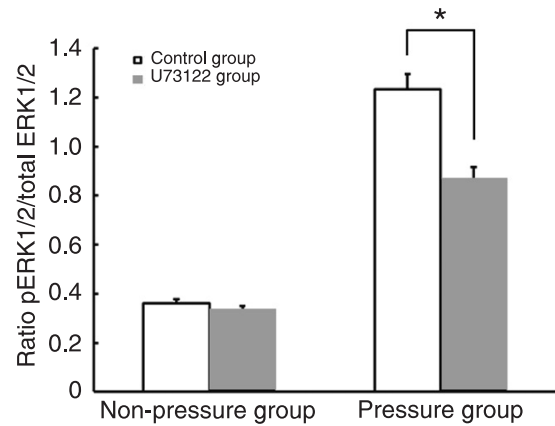

Figure 5. Effect of U73122 on the expression and phosphorylation of PLCY1, Src, MEK1/2, and ERK1/2 under conditions of periodic mechanical stress. After pretreatment with DMSO or PLCY1 inhibitor (U73122), rat chondrocytes were cultured in vitro for $1 \mathrm{~h}$ under static conditions or conditions of periodic mechanical stress. The expression and phosphorylation levels of PLCY1, Src, MEK1/2, and ERK1/2 were detected by Western blotting. $A$, The total amount of PLCY1 served as the control. The images above the histogram are representative results of Western blotting. The phosphorylation levels of PLCY1-Tyr 783 in the U73122 group were significantly reduced relative to control in chondrocytes in response to periodic mechanical stress $\left(\mathrm{N}=5\right.$, ${ }^{*} \mathrm{P}<0.05$, Student unpaired $t$-test). $B$, The total amount of Src served as the control. The images above the histogram are representative results of Western blotting. The phosphorylation levels of Src-Tyr 418 in the U73122 group were not altered relative to those in the control group in chondrocytes in response to periodic mechanical stress $(\mathrm{N}=5, \mathrm{P}>0.05$, Student unpaired $t$-test). $C$, The total amount of MEK1/2 served as the control. The images above the histogram are representative results of Western blotting. The phosphorylation levels of MEK-Ser $217 / 221$ in the U73122 group were significantly decreased relative to those in the control group in chondrocytes in response to periodic mechanical stress $\left(N=5\right.$, ${ }^{*} P<0.05$, Student unpaired $t$-test). $D$, The total amount of ERK1/2 served as the control. The images above the histogram are representative results of Western blotting. The phosphorylation levels of ERK1/2-Thr $202 / \mathrm{Tyr}^{204}$ in the U73122 group were significantly decreased relative to those in the control group in chondrocytes in response to periodic mechanical stress ( $N=5,{ }^{*} P<0.05$, Student unpaired $t$-test). 

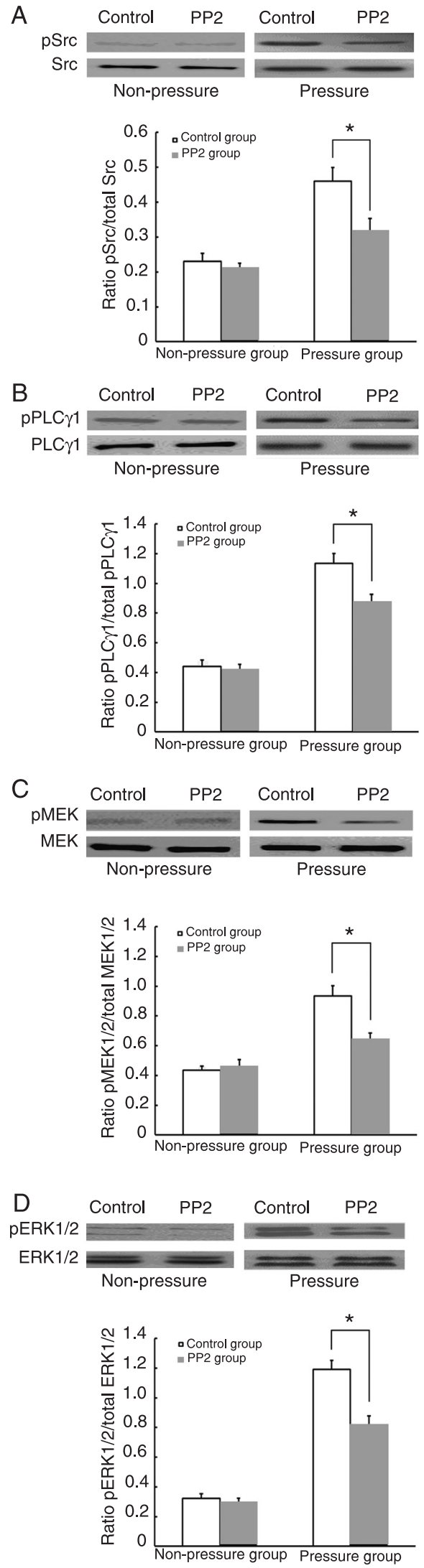
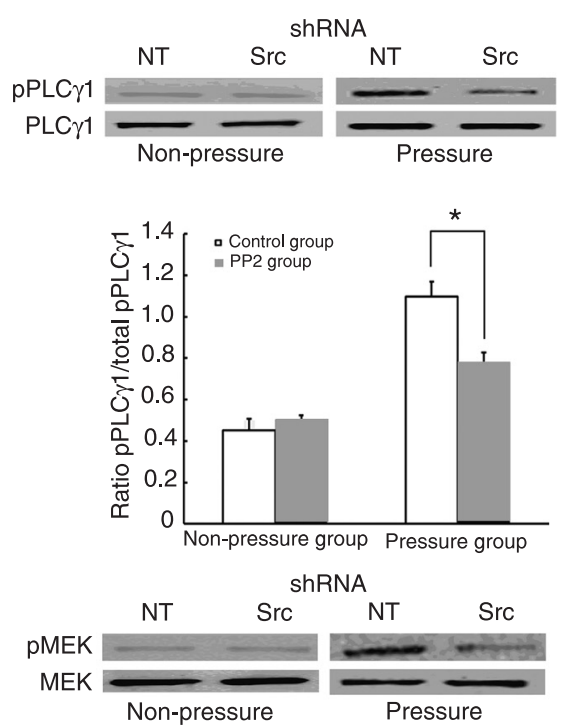

n-pressure
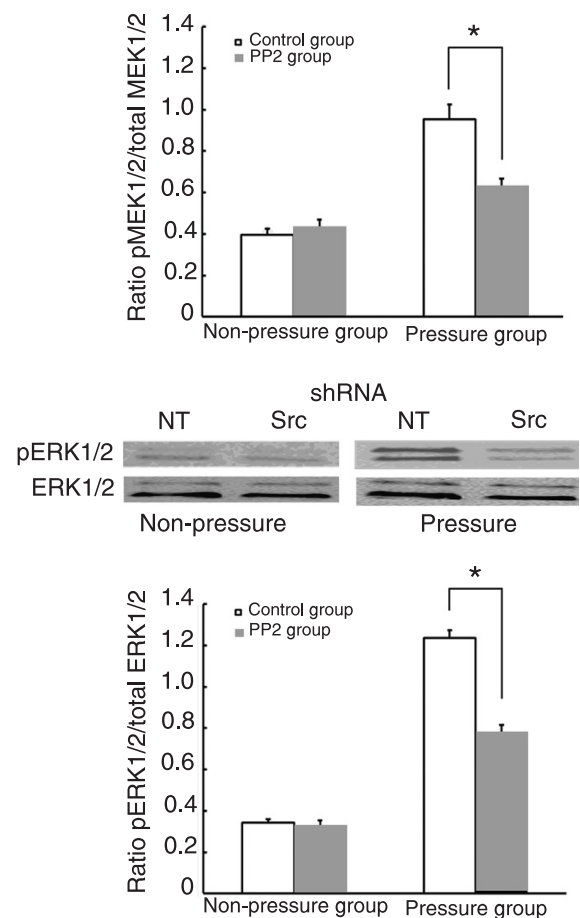

Figure 6. Effect of PP2 and Src shRNA on the expression and phosphorylation of Src, PLCY1, MEK1/2, and ERK1/2 under conditions of periodic mechanical stress. After pretreatment with DMSO or Src inhibitor (PP2), or Src shRNA or non-targeting (NT) sequence, rat chondrocytes were cultured in vitro for $1 \mathrm{~h}$ under static conditions or conditions of periodic mechanical stress. The expression and phosphorylation levels of Src $(A), \operatorname{PLC} 1$ 1 $(B), \operatorname{MEK} 1 / 2(C)$, and ERK1/2 $(D)$ were detected by Western blotting. For each Western blot, the corresponding total amount of each signaling protein served as a control. The images above the histogram are representative results of several independent Western blots. The phosphorylation levels of $\mathrm{Src}-\mathrm{Tyr}^{418}$ in the PP2 groups were significantly reduced compared to those in the control groups in chondrocytes in response to periodic mechanical stress $\left(N=5,{ }^{*} P<0.05\right.$, Student unpaired $t$-test). The phosphorylation levels of PLCY1-Tyr ${ }^{783}$, MEK-Ser217/221 and ERK1/2$\mathrm{Thr}^{202 / T y r} 204$ in the PP2 and Src shRNA groups were significantly reduced compared to those in the control groups in chondrocytes in response to periodic mechanical stress $\left(N=5,{ }^{*} P<0.05\right.$, Student unpaired $t$-test). 
phosphorylation level of ERK1/2. Thus, PD98059 was used to attenuate ERK1/2 activation, consistent with previously published studies regarding signaling transduction $(25,34)$. The present study showed that periodic mechanical stress promotes chondrocyte proliferation, AGC and Col2 gene expression, and also activates ERK1/2. Pretreatment with the MEK selective inhibitor, PD98059, or ERK1/2 shRNA attenuated periodic mechanical stress-induced chondrocyte proliferation, AGC and Col2 gene expression and ERK1/2 activation. These results provide definitive evidence to support the participation of MEK1/2-ERK1/2 signaling in the periodic mechanical stress-initiated mitogenic response and matrix synthesis in chondrocytes.

PLCY1 is one of the upstream mediators of ERK1/2, which presents the activity of serine threonine kinases. Phosphorylation of PLCY1-Tyr ${ }^{783}$ plays an important role in the signal transduction of cell proliferation and migration by mediating the downstream signaling proteins, including ERK1/2 $(16,22)$. Husain et al. (35) confirmed that activation of PLCY1 is required for vascular endothelial growth factor receptor-2 (VEGFR-2)induced endothelial cell proliferation in vitro. It has been demonstrated that in our previous study (12) periodic mechanical stress can up-regulate the phosphorylation of PLCY1 in chondrocytes, leading to cell area expansion and migration. Given the broad spectrum of roles in which PLCY1 is involved, it is possible that PLCY1 is associated with other mechanical stress responses of chondrocytes, including proliferation and matrix synthesis. It is well known that activated PLCY1 can promote the release of $\mathrm{Ca}^{2+}$ in the endoplasmic reticulum, which enhances the concentration of intracellular $\mathrm{Ca}^{2+}$ from many cell types. Furthermore, our previous study demonstrated that periodic mechanical stress could increase the concentration of intracellular free $\mathrm{Ca}^{2+}$, which leads to the up-regulation of chondrocyte proliferation and ultimately to matrix synthesis (36). Therefore, we speculate that there may be some causal relationship between the activation of PLCY1 and chondrocyte proliferation and matrix synthesis. The results of the present study showed that periodic mechanical stress not only causes chondrocyte proliferation, AGC and Col2 gene expression, but also activates PLCY1-Tyr ${ }^{783}$. We showed here that inhibition of PLCY1 abrogated cell proliferation, AGC and Col2 gene expression in chondrocytes in response to cyclic mechanical stress. Additionally, the up-regulated phosphorylation level of MEK1/2 and ERK1/2 initiated by periodic mechanical stimulation is attenuated as well as when the activity of PLCY1 is inhibited. These data support the view that PLCY1, which is located upstream of MEK1/2ERK1/2, is responsible for the up-regulation of chondrocyte proliferation, AGC and Col2 gene expression initiated by periodic mechanical stress.

Src is a member of the Src family, which shows the activ-

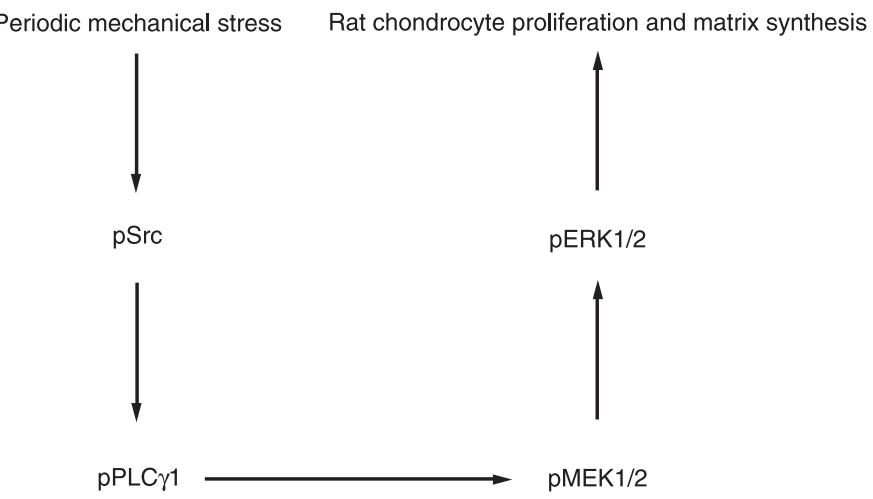

Figure 7. Schematic diagram of the signal transduction pathway of rat chondrocyte proliferation and matrix synthesis induced by periodic mechanical stress.

ity of tyrosine kinase. As part of a mechanosensory protein complex, Src mediates the cellular response to mechanical stimuli in numerous non-chondrocytic cell types $(23,37)$. In particular, Chaturvedi et al. $(14,15)$ have shown that, in the study of proliferation and migration of human Caco-2 intestinal epithelial cells mediated by rhythmic mechanical deformation, Src is a proximal kinase in the regulation of both proliferation and migration. Furthermore, our previous studies have shown that activated $\mathrm{Src}$ regulates periodic mechanical stress-initiated non-mitogenic effects in chondrocytes (12). In the present study, we found that periodic mechanical stress promotes chondrocyte proliferation, AGC and $\mathrm{Col} 2$ gene expression, with the phosphorylation level of Src-Tyr ${ }^{418}$ sites being also significantly increased. To evaluate the role of Src following cyclic mechanical stress, chondrocytes were incubated with the selective inhibitor PP2 or shRNA targeted to Src. The present data showed that inhibition of Src activity abrogated cell proliferation, AGC and Col2 gene expression, and attenuated PLCY1, ERK1/2 activation in chondrocytes subjected to cyclic mechanical stimuli. These observations further indicate the essential direct role of Src as a PLCY1, ERK1/2 upstream signaling mediator, in periodic mechanical stress-induced mitogenicity by placing it within the context of a mitogenic signal cascade also involving PLCY1 and ERK1/2. Our results demonstrate clearly that periodic mechanical stress stimulates Src activation, which subsequently regulates the activities of PLCY1 and ERK1/2 to promote chondrocyte proliferation and matrix synthesis under conditions of periodic mechanical stress.

Periodic mechanical stress is advantageous to promote the functions of seed cells and enhance the quality of tissueengineered cartilage so that it plays a crucial role in articular cartilage repair and regeneration, and is thus a focus of tissue engineering. A prerequisite for articular cartilage tissue engineering is an understanding of the signal transduction pathways involved in physiologic mechanical compression. 
Our previous study (12) has found that periodic mechanical stress promotes chondrocyte area expansion and migration through the Src-PLCY1-ERK1/2 signaling pathway. Our present results, taken together, precisely indicate that SrcPLCY1-ERK1/2 is one of the important signal transduction pathways in the processes of chondrocyte proliferation and matrix synthesis induced by periodic mechanical stress. It has come to light that chondrocyte proliferation and migration function as major determinants directly affecting the quality of tissue-engineered cartilage $(5,38,39)$. Therefore, Src-PLCY1-ERK1/2 is one of the crucial signaling pathways that improve the quality of tissue-engineered cartilage in two different aspects under conditions of periodic mechanical stress. The results of our previous (12) and present study provide a strong justification for further investigations regard-

\section{References}

1. Hirano $\mathrm{Y}$, Ishiguro $\mathrm{N}$, Sokabe $\mathrm{M}$, Takigawa $\mathrm{M}$, Naruse $\mathrm{K}$. Effects of tensile and compressive strains on response of a chondrocytic cell line embedded in type I collagen gel. $J$ Biotechnol 2008; 133: 245-252.

2. Wang PY, Chow HH, Lai JY, Liu HL, Tsai WB. Dynamic compression modulates chondrocyte proliferation and matrix biosynthesis in chitosan/gelatin scaffolds. J Biomed Mater Res B Appl Biomater 2009; 91: 143-152.

3. Wolf A, Ackermann B, Steinmeyer J. Collagen synthesis of articular cartilage explants in response to frequency of cyclic mechanical loading. Cell Tissue Res 2007; 327: 155-166.

4. Akimoto $\mathrm{T}$, Kawanishi M, Ushida $\mathrm{T}$. [Mechanical stress and tissue engineering]. Clin Calcium 2008; 18: 1313-1320.

5. Spiteri CG, Young EW, Simmons CA, Kandel RA, Pilliar RM. Substrate architecture and fluid-induced shear stress during chondrocyte seeding: role of alpha5beta1 integrin. Biomaterials 2008; 29: 2477-2489.

6. Isenovic ER, Kedees MH, Haidara MA, Trpkovic A, Mikhailidis DP, Marche P. Involvement of ERK1/2 kinase in insulin- and thrombin-stimulated vascular smooth muscle cell proliferation. Angiology 2010; 61: 357-364.

7. Severin S, Ghevaert C, Mazharian A. The mitogen-activated protein kinase signaling pathways: role in megakaryocyte differentiation. J Thromb Haemost 2010; 8: 17-26.

8. Lemieux E, Boucher MJ, Mongrain S, Boudreau F, Asselin C, Rivard N. Constitutive activation of the MEK/ERK pathway inhibits intestinal epithelial cell differentiation. Am J Physiol Gastrointest Liver Physiol 2011; 301: G719-G730.

9. Ryan JA, Eisner EA, DuRaine G, You Z, Reddi AH. Mechanical compression of articular cartilage induces chondrocyte proliferation and inhibits proteoglycan synthesis by activation of the ERK pathway: implications for tissue engineering and regenerative medicine. J Tissue Eng Regen Med 2009; 3: 107-116.

10. Hung CT, Henshaw DR, Wang CC, Mauck RL, Raia F, Palmer $\mathrm{G}$, et al. Mitogen-activated protein kinase signaling in bovine articular chondrocytes in response to fluid flow does not require calcium mobilization. J Biomech 2000; 33: 73-80.

11. Fitzgerald JB, Jin M, Chai DH, Siparsky P, Fanning P, Grodzinsky AJ. Shear- and compression-induced chondrocyte ing chondrocyte mechanobiology under periodic mechanical stress and how to improve the quality of tissue-engineered cartilage (Figure 7).

Src, PLCY1, MEK1/2, and ERK1/2, as important mediators of periodic mechanical stress-induced mitogenic effects in chondrocytes as described above, are not the sole signaling proteins. Thus, whether or not other signaling molecules are involved deserves further study. In particular, how Src is initially activated in response to periodic mechanical stress in chondrocytes awaits further investigation.

\section{Acknowledgments}

Research supported by the National Natural and Science Foundation (\#30876338 and \#81000800). transcription requires MAPK activation in cartilage explants. J Biol Chem 2008; 283: 6735-6743.

12. Nong L, Yin G, Ren K, Tang J, Fan W. Periodic mechanical stress enhances rat chondrocyte area expansion and migration through Src-PLCgamma1-ERK1/2 signaling. Eur J Cell Biol 2010; 89: 705-711.

13. Perera PM, Wypasek E, Madhavan S, Rath-Deschner B, Liu J, Nam J, et al. Mechanical signals control SOX-9, VEGF, and c-Myc expression and cell proliferation during inflammation via integrin-linked kinase, B-Raf, and ERK1/2dependent signaling in articular chondrocytes. Arthritis Res Ther 2010; 12: R106.

14. Chaturvedi LS, Marsh HM, Shang X, Zheng Y, Basson MD. Repetitive deformation activates focal adhesion kinase and ERK mitogenic signals in human Caco-2 intestinal epithelial cells through Src and Rac1. J Biol Chem 2007; 282: 14-28.

15. Chaturvedi LS, Gayer CP, Marsh HM, Basson MD. Repetitive deformation activates Src-independent FAK-dependent ERK motogenic signals in human Caco-2 intestinal epithelial cells. Am J Physiol Cell Physiol 2008; 294: C1350-C1361.

16. Shen E, Fan J, Chen R, Yee SP, Peng T. Phospholipase Cgamma1 signalling regulates lipopolysaccharide-induced cyclooxygenase-2 expression in cardiomyocytes. J Mol Cell Cardiol 2007; 43: 308-318.

17. Qi YX, Qu MJ, Yan ZQ, Zhao D, Jiang XH, Shen BR, et al. Cyclic strain modulates migration and proliferation of vascular smooth muscle cells via Rho-GDlalpha, Rac1, and p38 pathway. J Cell Biochem 2010; 109: 906-914.

18. Kingsley K, Huff JL, Rust WL, Carroll K, Martinez AM, Fitchmun $\mathrm{M}$, et al. ERK1/2 mediates PDGF-BB stimulated vascular smooth muscle cell proliferation and migration on laminin-5. Biochem Biophys Res Commun 2002; 293: 10001006.

19. Hattori T, Ara T, Wang P. Participation of tyrosine kinase and phospholipase Cgamma in isradipine-induced proliferation of cultured human gingival fibroblasts. Eur J Med Res 2005; 10: $543-546$

20. Heo JS, Lee YJ, Han HJ. EGF stimulates proliferation of mouse embryonic stem cells: involvement of $\mathrm{Ca}^{2+}$ influx and p44/42 MAPKs. Am J Physiol Cell Physiol 2006; 290: 
C123-C133.

21. El Hiani Y, Ahidouch A, Lehen'kyi V, Hague F, Gouilleux F, Mentaverri R, et al. Extracellular signal-regulated kinases 1 and 2 and TRPC1 channels are required for calcium-sensing receptor-stimulated MCF-7 breast cancer cell proliferation. Cell Physiol Biochem 2009; 23: 335-346.

22. Liu M, Xu J, Liu J, Kraw ME, Tanswell AK, Post M. Mechanical strain-enhanced fetal lung cell proliferation is mediated by phospholipase $\mathrm{C}$ and $\mathrm{D}$ and protein kinase C. Am J Physiol 1995; 268: L729-L738.

23. Morita N, lizuka K, Murakami T, Kawaguchi H. N-terminal kinase, and $\mathrm{c}-\mathrm{Src}$ are activated in human aortic smooth muscle cells by pressure stress. Mol Cell Biochem 2004; 262: 71-78.

24. Chaturvedi LS, Marsh HM, Basson MD. Src and focal adhesion kinase mediate mechanical strain-induced proliferation and ERK1/2 phosphorylation in human $\mathrm{H} 441$ pulmonary epithelial cells. Am J Physiol Cell Physiol 2007; 292: C1701C1713.

25. Gemba T, Valbracht J, Alsalameh S, Lotz M. Focal adhesion kinase and mitogen-activated protein kinases are involved in chondrocyte activation by the $29-\mathrm{kDa}$ amino-terminal fibronectin fragment. J Biol Chem 2002; 277: 907-911.

26. Seguin CA, Bernier SM. TNFalpha suppresses link protein and type II collagen expression in chondrocytes: Role of MEK1/2 and NF-kappaB signaling pathways. J Cell Physiol 2003; 197: 356-369.

27. Yue HT, Fan WM, Ma YM. Tissue engineered cartilage in response to diferent cyclic pressure. Chin J Orthop Trauma 2007; 9: 661-664.

28. Zhang GC, Fan WM, Ma YM. Effects of cyclic pressure at different frequendes on the construction of tissue-engineering cartilage. Chin J Exp Surg 2007; 24: 677-678.

29. Wang P, Cao X, Nagel DJ, Yin G. Activation of ASK1 during reperfusion of ischemic spinal cord. Neurosci Lett 2007; 415: 248-252.

30. Li KW, Wang AS, Sah RL. Microenvironment regulation of extracellular signal-regulated kinase activity in chondro- cytes: effects of culture configuration, interleukin-1, and compressive stress. Arthritis Rheum 2003; 48: 689-699.

31. Yin G, Zheng Q, Yan C, Berk BC. GIT1 is a scaffold for ERK1/2 activation in focal adhesions. $J$ Biol Chem 2005; 280: 27705-27712.

32. Vincent TL, Hermansson MA, Hansen UN, Amis AA, Saklatvala J. Basic fibroblast growth factor mediates transduction of mechanical signals when articular cartilage is loaded. Arthritis Rheum 2004; 50: 526-533.

33. Yin J, Yu FS. ERK1/2 mediate wounding- and G-proteincoupled receptor ligands-induced EGFR activation via regulating ADAM17 and HB-EGF shedding. Invest Ophthalmol Vis Sci 2009; 50: 132-139.

34. Francis H, Glaser S, Ueno Y, Lesage G, Marucci L, Benedetti $A$, et al. CAMP stimulates the secretory and proliferative capacity of the rat intrahepatic biliary epithelium through changes in the PKA/Src/MEK/ERK1/2 pathway. J Hepatol 2004; 41: 528-537.

35. Husain D, Meyer RD, Mehta M, Pfeifer WM, Chou E, Navruzbekov G, et al. Role of c-Cbl-dependent regulation of phospholipase Cgamma1 activation in experimental choroidal neovascularization. Invest Ophthalmol Vis Sci 2010; 51: 6803-6809.

36. Fan WM, Cai J. The explore of the effects of cyclic pressure on construction of tissue engineer cartilage and its mechanism. Chin J Exp Surg 2006; 23: 977-979.

37. Lawler K, O'Sullivan G, Long A, Kenny D. Shear stress induces internalization of E-cadherin and invasiveness in metastatic oesophageal cancer cells by a Src-dependent pathway. Cancer Sci 2009; 100: 1082-1087.

38. Spiteri CG, Pilliar RM, Kandel RA. Substrate porosity enhances chondrocyte attachment, spreading, and cartilage tissue formation in vitro. J Biomed Mater Res A 2006; 78: 676-683.

39. Tsai YH, Chen CW, Lai WF, Tang JR, Deng WP, Yeh SD, et al. Phenotypic changes in proliferation, differentiation, and migration of chondrocytes: $3 \mathrm{D}$ in vitro models for joint wound healing. J Biomed Mater Res A 2010; 92: 1115-1122. 